\title{
Application of a treatment planning system-assisted large-aperture computed tomography simulator to percutaneous biopsy: initial experience of a radiation therapist
}

Journal of International Medical Research 49 (I) $1-12$

(C) The Author(s) 2021 Article reuse guidelines: sagepub.com/journals-permissions DOI: I0.I I77/0300060520983 |4 I journals.sagepub.com/home/imr @SAGE

\author{
Xiaoyi Lin ${ }^{\mathrm{l} *}$, Liqin $\mathrm{Ma}^{2}$, , Kaixin $\mathrm{Du}^{\mathrm{I}}$, \\ Junqiang Hong', Shuiying Luo', Youqun Lai', \\ Yongliang Dai ${ }^{1}$ and Xiangquan Kong ${ }^{1}$ (D)
}

\begin{abstract}
Objective: To evaluate the application of treatment planning system (TPS)-assisted largeaperture computed tomography (CT) simulator to percutaneous biopsy.

Methods: This retrospective study enrolled patients that underwent TPS-assisted large-aperture CT simulator-guided percutaneous biopsy from November 2018 to December 2019. Retrospective analyses of puncture accuracy were compared using paired $t$-test and a Wilcoxon rank sum test. The risk factors for puncture accuracy and complications were identified.
\end{abstract}

Results: A total of 38 patients were included in this study. There were no significant differences between the planned and actual puncture depth and angle. Pulmonary puncture was significantly associated with the accuracy of the puncture angle. The diagnostic rate of malignancy was $76 \%$ (29 of 38), of which 20 of 25 patients were in the group initially diagnosed with unconfirmed lesions and nine of 13 patients were in the group of treated patients that needed additional pathological analyses. For patients that underwent a pulmonary biopsy, 12 had minor pneumothorax and three suffered needle track bleeding. No other complications were observed.

\footnotetext{
'Department of Radiation Oncology, Xiamen Humanity Hospital Fujian Medical University, Xiamen, Fujian Province, China

${ }^{2}$ Department of Radiation Oncology, Fujian Medical University Cancer Hospital, Fuzhou, Fujian Province, China
}

\footnotetext{
*These authors contributed equally to this work.

Corresponding author:

Xiangquan Kong, Department of Radiation Oncology,

Xiamen Humanity Hospital Fujian Medical University,

3777 Xianyue Road, Huli District, Xiamen 36I006, Fujian Province, China.

Email: kongxiangquan2020@aliyun.com
} 
Regression analyses indicated a significant correlation between puncture angle and the incidence of pneumothorax.

Conclusion: TPS-assisted large-aperture CT simulator may improve the percutaneous biopsy procedure by combining the advantages of radiotherapy specialties with computer targeting.

\section{Keywords}

Treatment planning system-assisted, percutaneous biopsy, computed tomography simulatorguided, puncture accuracy

Date received: 29 June 2020; accepted: 2 December 2020

\section{Introduction}

Pathological results, as the standard for tumour diagnosis, provide vital information for qualitative diagnosis, treatment guidance and prognostic evaluation. Computed tomography (CT)-guided percutaneous biopsy, which has been extensively used in the clinic for pulmonary and abdominal lesions, plays an important role in obtaining tissue samples and making precise pathological diagnoses. Recently, a considerable body of literature has focused on improving the accuracy and efficiency of this puncture process, through the application of the puncture site-down positioning technique, automatic path proposal computation or large-core needles. ${ }^{1-3}$ Nevertheless, several major issues, such as inaccurate positioning, repeated adjustment of the puncture route and the risk of serious postoperative complications, remain unresolved. ${ }^{4-7}$

Considering that CT-guided percutaneous biopsy is generally performed by interventional radiologists or a thoracic surgeon, the present research aimed to exploit the advantages of a radiotherapy simulator and a treatment planning system (TPS) in a department of radiation oncology to compensate for technical defects. Since 2018, the clinical application of a TPS-assisted largeaperture CT simulator to percutaneous biopsy has been investigated and the initial experience is summarized in this current study, with the aim of offering new insights into the improvement of puncture operation and biopsy accuracy.

\section{Patients and methods}

\section{Patient population}

This retrospective study enrolled consecutive patients that underwent TPS-assisted large-aperture CT simulator-guided percutaneous biopsy at the Department of Radiation Oncology, Xiamen Humanity Hospital Fujian Medical University, Xiamen, Fujian Province, China between November 2018 and December 2019. The clinical data were retrospectively analysed. Inclusion criteria were as follows: (i) initially diagnosed with unconfirmed lesions (i.e. lesions were initially detected by imaging but qualitative diagnosis was unclear) or treated patients that needed additional pathology information for treatment guidance; (ii) available puncture path information; (iii) generally good health, Karnofsky Performance Score (KPS) $\geq 70$, tolerable to puncture procedure. Exclusion criteria were as follows: (i) poor general condition or cachexia, KPS $<70$, or intolerable to puncture procedure; (ii) coagulation dysfunction 
with haemorrhagic tendencies; (iii) severe cardiocerebrovascular disease; (iv) severe infection; (v) superficial lesions could be used to obtain tissue specimens without imaging or B ultrasound-guided puncture; (iv) compulsive position due to spinal disease or other reasons.

This study was approved by the Ethics Committee of Xiamen Humanity Hospital Fujian Medical University (no. XMHA2020004) in March 2020 and undertaken in accordance with the Declaration of Helsinki. All patients provided written informed consent before puncture. Verbal consent for publication was obtained from patients whose images are contained in the manuscript. In order to protect their identities, the details of all patients were deidentified.

\section{Puncture procedures}

The puncture workflow is shown in Figure 1. The preoperative preparation involved air sterilization of the CT simulator room, confirmation of the puncture supplies and rescue medicines, and measurement of the life indications of each patient. The following equipment was used: a Philips 16-multislice spiral large-aperture CT simulator (Philips Healthcare, Best, the Netherlands); a movable three-dimensional laser positioning system (Philips Healthcare); an immobilization device (vacuum fixation mat or prone board; Klarity, Guangzhou, China); an Eclipse 13.5 planning system (Varian Medical Systems, Palo Alto City, CA, USA); and a Bard 17G coaxial biopsy needle (Bard, Murray Hill, NJ, USA). For the immobilization and CT scan, the optimum position was selected for patients according to the location of each target lesion and a vacuum fixation mat or prone board was applied for fixing the patients in comfortable positions prior to biopsy. Then, location points were selected and spherical metal markers were pasted on the patients' surface. Cross hatchings were marked following the

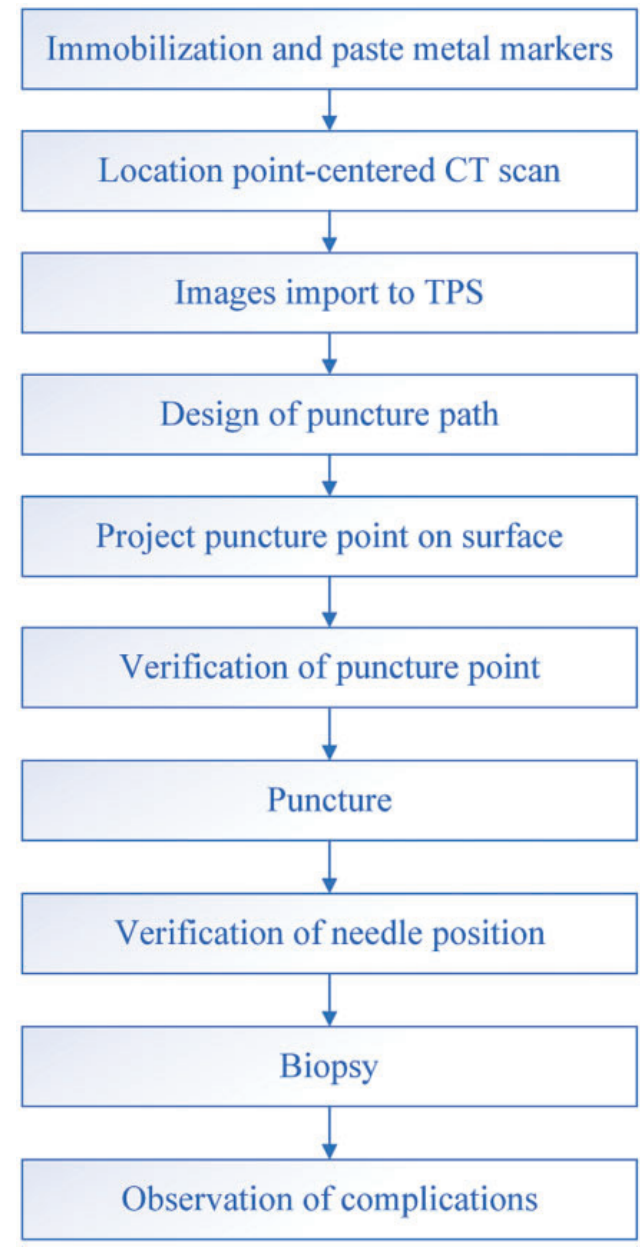

Figure I. Flow diagram of treatment planning system (TPS)-assisted large-aperture computed tomography (CT) simulator guided percutaneous biopsy.

laser lines and extension lines on the vacuum fixation mat or prone immobilization device were drawn to determine the relative position of the lesion. Location point-centred CT scan was then subsequently performed to a layer thickness of $3 \mathrm{~mm}$ and the images were transmitted to the radiotherapy server. For determination of the puncture path, CT images were imported into Varian TPS (Varian Medical Systems) and the origin was set on TPS. To design the optimum puncture path, 
the maximum cross-section level of the target lesion was taken when possible, avoiding bones and large blood vessels, and to ensure that normal tissue was pierced as infrequently as possible. The puncture target, known as the gross tumour volume (GTV), was delineated by a radiation therapist (X.K.) and a new plan was created by the radiotherapy physicist (Y.L.) according to the planned path. Subsequently, the source skin distance (SSD) was changed to $100 \mathrm{~cm}(\mathrm{SSD}=100 \mathrm{~cm})$ and the relative coordinates ( $\mathrm{X}, \mathrm{Y}$ and $\mathrm{Z}$ values) of the location point and the puncture point were recorded. The puncture direction and depth were also recorded. With the assistance of the laser positioning system, the puncture point based on the relative coordinates was accurately located onto the patient's body surface. The above operations were checked by two technicians before puncture. Verification of the puncture point involved the puncture points being marked and axial scans were performed to verify the location of each puncture point, avoiding set-up error. For the percutaneous biopsy, following routine sterilization and local anaesthesia administration, a coaxial biopsy needle was punctured into the proximal region of the lesion based on the planned direction and depth (calibrated by laser line and protractor). Additionally, the location of the biopsy needle was verified by CT scan (for adjustment if necessary). Two or three satisfactory specimens were collected in a specimen bottle with formalin and sent for pathological examination. The skin of the puncture region was then disinfected and bound up postoperatively. A CT scan of the puncture site and adjacent areas was performed to confirm whether there were signs of pneumothorax or bleeding. Close observation for $6 \mathrm{~h}$ was required for each patient enrolled in the study.

\section{Outcome measures}

The planned and actual puncture direction and depth were recorded for comparison separately. $\Delta \mathrm{H}$ was defined as the difference between the planned and actual puncture depth; and $\Delta \alpha$ was defined as the difference between the planned and actual puncture angle. Other indices including puncture time, frequency of needle adjustment, pathological results and complications were recorded.

\section{Statistical analyses}

All statistical analyses were performed using IBM SPSS Statistics for Windows, Version 23.0 (IBM Corp., Armonk, NY, USA). The parameters of puncture accuracy were analysed using a paired $t$-test and Wilcoxon rank sum test. Regression analysis was used to identify the risk factors of puncture complications. The workflow was drawn in Microsoft Visio 2019 (Microsoft, Renton, WA, USA). A $P$-value $<0.05$ was considered statistically significant.

\section{Results}

This retrospective analysis included 38 patients (25 males and 13 females) that underwent TPS-assisted large-aperture CT simulator-guided percutaneous biopsy. The median age of the study cohort was 62 years (range, 32-87 years). Percutaneous lung biopsy was performed in 34 patients, abdominal lesion biopsy was performed in three patients (including liver, pancreas and adrenal gland) and there was one patient that underwent a deep supraclavicular lymph node biopsy. Of the lesions, 36 of 38 $(95 \%)$ were solid. The median maximum diameter of the lesions was $3.8 \mathrm{~cm}$ (range, $1.1-10.1 \mathrm{~cm})$. The demographic and clinical characteristics of the patients are presented in Table 1.

The puncture time for each patient was within $20 \mathrm{~min}$. The entire cohort achieved a one-time successful biopsy. Thirty-four patients had CT scans four times, while the remaining four patients underwent one 
Table I. Clinical and demographic characteristics of the patients $(n=38)$ that were enrolled in a retrospective study investigating the clinical application of a treatment planning system-assisted large-aperture computed tomography simulator to percutaneous biopsy.

\begin{tabular}{|c|c|}
\hline Characteristic & $\begin{array}{l}\text { Study cohort } \\
n=38\end{array}$ \\
\hline \multicolumn{2}{|l|}{ Sex } \\
\hline Male & $25(66)$ \\
\hline Female & $13(34)$ \\
\hline \multicolumn{2}{|l|}{ Age, years } \\
\hline Median & 62 \\
\hline Range & $32-87$ \\
\hline \multicolumn{2}{|l|}{ Puncture purpose } \\
\hline Initial unconfirmed lesion & $25(66)$ \\
\hline Therapeutic evaluation & $8(2 I)$ \\
\hline $\begin{array}{l}\text { Gene detection after } \\
\text { primary diagnosis }\end{array}$ & $5(13)$ \\
\hline \multicolumn{2}{|l|}{ Lesion location } \\
\hline Superior lobe of left lung & II (29) \\
\hline Inferior lobe of left lung & $6(16)$ \\
\hline Superior lobe of right lung & $10(26)$ \\
\hline Middle lobe of right lung & $4(1 \mathrm{I})$ \\
\hline Inferior lobe of right lung & $2(5)$ \\
\hline Left pleura & I (3) \\
\hline $\begin{array}{l}\text { Right supraclavicular } \\
\text { lymph node }\end{array}$ & I (3) \\
\hline $\begin{array}{l}\text { Abdomen (liver, pancreas } \\
\text { and adrenal gland) }\end{array}$ & $3(8)$ \\
\hline \multicolumn{2}{|l|}{ Lesion character } \\
\hline Solid & $36(95)$ \\
\hline Frosted glass & I (3) \\
\hline Solid-cystic & I (3) \\
\hline \multicolumn{2}{|l|}{ Maximum diameter, $\mathrm{cm}$} \\
\hline Median & 3.8 \\
\hline Range & $1.1-10.1$ \\
\hline
\end{tabular}

Data presented as median (range) or $n$ of patients (\%).

additional CT scan due to slight deviation adjustments. The actual puncture depths were close to the planned puncture depths and there was no significant difference between the mean \pm SD planned and actual puncture depths $(5.34 \pm 2.01 \mathrm{~cm}$ versus $5.28 \pm 1.95 \mathrm{~cm}$, respectively) (Table 2 ). The mean $\pm \mathrm{SD}$ deviation of the puncture depth $\Delta \mathrm{H}$ was $0.37 \pm 0.32 \mathrm{~cm}$, which indicated the
Table 2. Puncture accuracy in patients $(n=38)$ that were enrolled in a retrospective study investigating the clinical application of a treatment planning system-assisted large-aperture computed tomography simulator to percutaneous biopsy.

\begin{tabular}{lll}
\hline Parameter & Planned & Actual \\
\hline $\begin{array}{l}\text { Puncture depth, cm } \\
\text { Puncture angle, }{ }^{\circ}\end{array}$ & $5.34 \pm 2.01$ & $5.28 \pm 1.95$ \\
\hline
\end{tabular}

Data presented as mean $\pm S D$ or median.

No significant between group differences $(P \geq 0.05)$; paired $t$-test and Wilcoxon rank sum test.

precision of the puncture depth. With regard to the puncture angle, there was no significant difference in the median planned and actual angles, which were $90^{\circ}$ and $88^{\circ}$, respectively, demonstrating the high reproducibility of this technique.

Data presented in Table 3 show that the deviations in the puncture depth and angle were well controlled regardless of the size and location of the lesions. Although no significant differences were detected in the puncture accuracy between the different lesion size subgroups, $\Delta \mathrm{H}$ decreased with increasing lesion size. In addition, $\Delta \alpha$ in the superior lobe of the lung was significantly smaller than that in the middle or lower lobes $\left(\chi^{2}=-2.129, P=0.033\right)$, suggesting that the pulmonary puncture location had an effect on the accuracy of the puncture angle. Examples of percutaneous pulmonary punctures are presented in Figures 2 and 3. An example of the prone position used during the biopsy procedure is show in Figure 4.

Biopsy specimens were successfully obtained in all 38 patients. The success rate of puncture was $100 \%$ (38 of 38 patients). As summarized in Table 4, the diagnostic rate of malignancy was $76 \%$ (29 of 38 patients), of which 20 of 25 patients were in the group initially diagnosed with unconfirmed lesions and nine of 13 patients were in the group of treated patients that needed additional pathological analyses. 
Table 3. Puncture accuracy in patients $(n=38)$ stratified according to the lesion size and puncture location.

\begin{tabular}{|c|c|c|c|c|c|c|}
\hline Variable & $\Delta \mathrm{H}, \mathrm{cm}$ & $\mathrm{Z} / \chi^{2}$ & $\begin{array}{l}\text { Statistical } \\
\text { analysis }^{\mathrm{a}}\end{array}$ & $\Delta \alpha,{ }^{\circ}$ & $\mathrm{Z} / \chi^{2}$ & $\begin{array}{l}\text { Statistical } \\
\text { analysis }^{\mathrm{a}}\end{array}$ \\
\hline Lesion size, $\mathrm{cm}$ & & 0.680 & NS & & 0.590 & NS \\
\hline$\leq 3(n=13)$ & $0.40 \pm 0.33$ & & & $3.77 \pm 3.49$ & & \\
\hline $3-5(n=19)$ & $0.35 \pm 0.30$ & & & $3.63 \pm 3.53$ & & \\
\hline$>5(n=6)$ & $0.33 \pm 0.40$ & & & $6.33 \pm 6.89$ & & \\
\hline Puncture location & & -0.599 & NS & & -2.129 & $P=0.033$ \\
\hline Superior lobe of lung $(n=2 \mathrm{I})$ & $0.34 \pm 0.27$ & & & $2.62 \pm 3.02$ & & \\
\hline Middle and inferior lobe $(n=12)$ & $0.46 \pm 0.4 \mathrm{I}$ & & & $5.42 \pm 3.80$ & & \\
\hline
\end{tabular}

Data presented as mean $\pm \mathrm{SD}$.

${ }^{a}$ Wilcoxon rank sum test; NS, no significant between group differences $(P \geq 0.05)$.

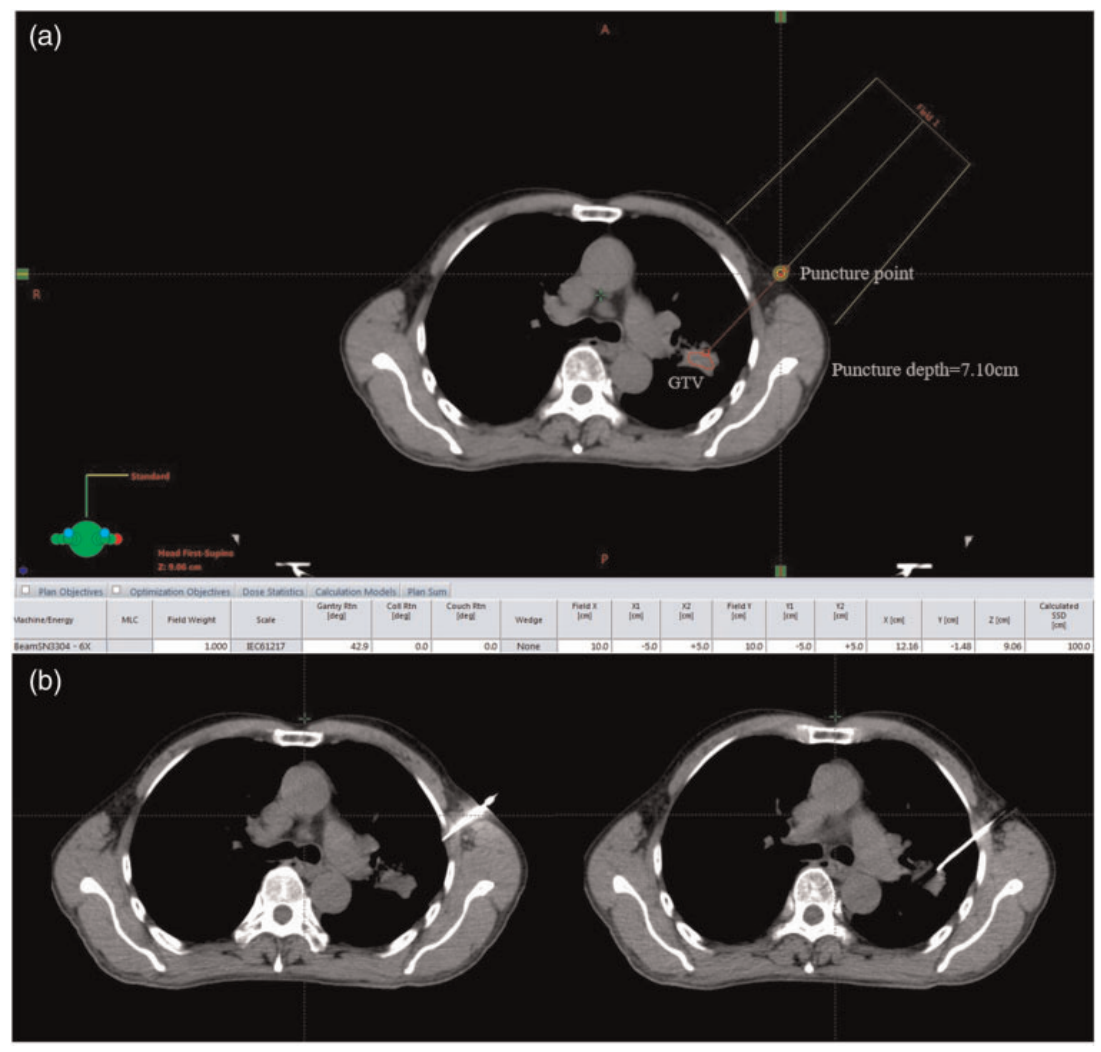

Figure 2. Representative computed tomography scans of an example of the biopsy planning. (a) A plan was created using the treatment planning system to design an optimum puncture path. The relative coordinates ( $\mathrm{X}, \mathrm{Y}$ and $\mathrm{Z}$ values) were generated automatically when the puncture point was determined. (b) The position of the biopsy needle was verified. GTV, gross tumour volume (i.e. puncture target). 


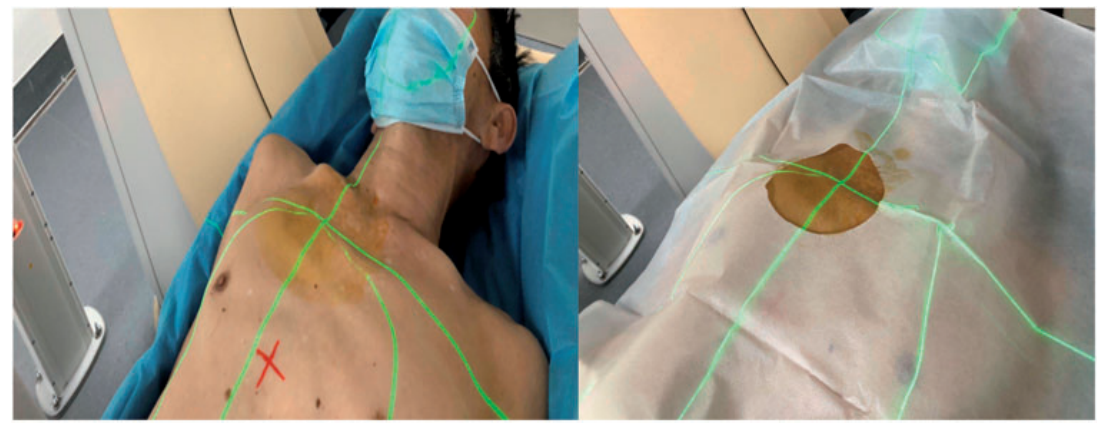

Figure 3. Representative photographs showing the patient positioning. With the help of the laser positioning system, a puncture point based on the relative coordinates was accurately located onto the body surface of the patient.

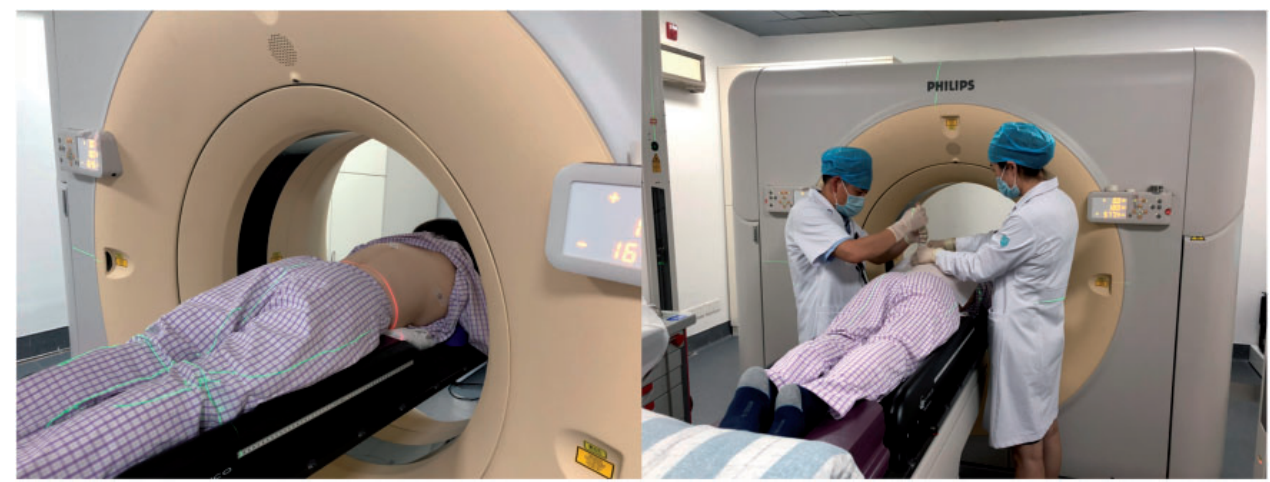

Figure 4. Representative photographs showing a patient in the prone position and the biopsy being undertaken.

Table 4. Pathological results of patients $(n=38)$ that were enrolled in a retrospective study investigating the clinical application of a treatment planning system-assisted large-aperture computed tomography simulator to percutaneous biopsy.

\begin{tabular}{lc}
\hline Pathological results & $n(\%)$ \\
\hline Adenocarcinoma & $16(42)$ \\
Squamous cell carcinoma & $6(16)$ \\
Small cell lung cancer & $2(5)$ \\
Metastatic sarcoma & $2(5)$ \\
Metastatic hepatocellular carcinoma & $1(3)$ \\
Malignant epithelial carcinoma & $2(5)$ \\
Inflammation & $7(18)$ \\
No malignancy & $2(5)$ \\
\hline
\end{tabular}

Among the 29 patients with malignant tumours, adenocarcinoma was confirmed in 16 patients, squamous cell carcinoma in six patients, small cell lung carcinoma in two patients, metastatic disease in three patients and the remaining two patients had epithelial carcinoma. The other nine patients were diagnosed with benign diseases.

Minor needle track bleeding was observed in three patients. For the 33 patients that underwent a pulmonary biopsy, 12 had a minor pneumothorax $(36 \%)$, of which two progressed to a massive pneumothorax at re-examination after $24-48 \mathrm{~h}(6 \%)$. Patients 
with a massive pneumothorax were treated successfully with closed thoracic drainage and the minor pneumothorax absorbed themselves without further intervention. No other complications, such as haemoptysis, haemothorax or subcutaneous haematoma, were found in the cohort. Moreover, no puncture-related complications, such as bleeding, infection or pancreatitis, were observed in the three patients that underwent an abdominal biopsy.

Based on the regression analyses (Table 5), a significant correlation was found between puncture angle and pneumothorax; the incidence rate of pneumothorax in punctures with an angle of $80-100^{\circ}$ was significantly higher than that with other angles (11 of 23 patients [ $48 \%$ ] versus one of 10 patients [ $10 \%$ ], respectively; $P=0.027$ ). Age, sex, reason for puncture, lesion size, lesion location, puncture depth and number of needle adjustments had no effect on pneumothorax. Although the findings were not significant, patients $\geq 65$ years or those with a puncture depth $\geq 5 \mathrm{~cm}$ were more susceptible to pneumothorax.

\section{Discussion}

In the era of individualized precision treatment, especially with the advancements in

Table 5. Regression analysis of factors affecting the incidence of pneumothorax in patients $(n=33)$ that underwent a pulmonary biopsy.

\begin{tabular}{|c|c|c|}
\hline Variable & $\begin{array}{l}\text { Pneumothorax/no } \\
\text { pneumothorax }\end{array}$ & $\begin{array}{l}\text { Statistical } \\
\text { analysis }\end{array}$ \\
\hline Sex & & NS \\
\hline Male $(n=21)$ & $9 / 12$ & \\
\hline Female $(n=12)$ & $3 / 9$ & \\
\hline Age, years & & NS \\
\hline$\geq 65(n=17)$ & $8 / 9$ & \\
\hline$<65(n=16)$ & $4 / 12$ & \\
\hline Puncture purpose & & NS \\
\hline Initial treatment $(n=22)$ & $9 / 13$ & \\
\hline Treated $(n=1 \mathrm{I})$ & $3 / 8$ & \\
\hline Lesion location & & NS \\
\hline Superior lobe of lung $(n=2 \mathrm{I})$ & $7 / 14$ & \\
\hline Middle and inferior lobe $(n=12)$ & $5 / 7$ & \\
\hline Lesion size, $\mathrm{cm}$ & & NS \\
\hline$\leq 3(n=4)$ & $2 / 2$ & \\
\hline $3-5(n=16)$ & $5 / 11$ & \\
\hline$>5(n=13)$ & $5 / 8$ & \\
\hline Puncture depth, $\mathrm{cm}$ & & NS \\
\hline$\geq 5(n=18)$ & $7 / 11$ & \\
\hline$<5(n=15)$ & $5 / 10$ & \\
\hline Puncture angle, ${ }^{\circ}$ & & $P=0.027$ \\
\hline $80-100(n=23)$ & $11 / 12$ & \\
\hline$<80$ or $>100(n=10)$ & $1 / 9$ & \\
\hline Needle adjustment & & NS \\
\hline Once $(n=4)$ & $1 / 3$ & \\
\hline No $(n=29)$ & $11 / 18$ & \\
\hline
\end{tabular}

Data presented as $n$ of patients.

NS, no significant association ( $P \geq 0.05)$. 
molecular targeted therapy and immunotherapy, pathology is still a prerequisite for further gene detection, exploration of drug resistance mechanisms and evaluation of patient conditions. ${ }^{8-10} \mathrm{CT}$-guided percutaneous biopsy, as an important solution for the acquisition of pathological information, is playing an increasingly critical role in clinical practice, ${ }^{11}$ which requires improved operability and accuracy. Unfortunately, there still exists defects in this process in terms of inaccurate positioning, low one-time success rate, heterogeneity of operators' experience and the poor compliance of some patients who are prone to serious complications. ${ }^{4-7}$ This is the first study to combine TPS and radiotherapy positioning systems with the puncture process and it has refined the success of this operation. The initial results from this current study suggest that this method has advantages in puncture accuracy and a higher success rate, with satisfactory safety. Thus, it has the potential to be an important contribution to the field of CTguided percutaneous biopsy.

The advantages of combining TPS with CT-guided percutaneous biopsy are summarized as follows: (i) the pre-puncture area (GTV) can be freely delineated by operators on TPS based on their clinical experience and the imaging features of lesions; (ii) regions of tumour liquefaction or necrosis can be avoided; and (iii) the detection rate can be improved. Furthermore, with accurate coordinate information conversion between TPS and a CT simulator system, the optimal puncture point is projected onto the patient's body surface based on relative coordinates, which are accurate to the millimetre. Therefore, the accuracy of positioning is greatly improved compared with the traditional method of using a standard lead point mesh and the restriction on the choice of puncture point is reduced to a certain extent.
Several attempts had been made to improve puncture accuracy, ${ }^{12,13}$ but these did not take into account the inevitable errors between actual operations and ideal pre-set parameters. This current study compared actual and planned puncture data and the results showed a good agreement between the two. There was no statistical difference between the actual and planned puncture depths $(5.28 \pm 1.95 \mathrm{~cm}$ versus $5.34 \pm 2.01 \mathrm{~cm}$, respectively) or angles $\left(88^{\circ}\right.$ versus $90^{\circ}$, respectively). The reason that the actual puncture depth was less than the planned depth might be due to different levels of skin retraction during the puncture process. Ultimately, the deviations were controlled within a satisfactory range and the one-time success rate reached $100 \%$, demonstrating that this technology was suitable for clinical application.

The success rate of CT-guided percutaneous biopsy reported varies between different techniques. For example, a previous study reported a puncture success rate of 98\% with template-assisted CT-guided percutaneous biopsy, of which the one-time success rate was $50 \% .^{12}$ Another study used an electromagnetic navigation system for CT-guided biopsy, reducing puncture time and radiation exposure. ${ }^{14}$ The success rate was $84.4 \%$ and the one-time success was only $58.3 \%{ }^{14}$ Similarly, large-core biopsy (10G needle) was successful in $88.6 \%{ }^{3}$ which was lower than that of the present study. As for puncture time, $20 \mathrm{~min}$ would not appear to be an advantage compared with other methods (range 9-34 $\min ),{ }^{3,13,14}$ but it was acceptable.

Puncture accuracy is thought to be associated with detection rate and it is influenced by multiple variables. For example, a previous study reported on 123 patients with pulmonary nodules and found that diagnostic accuracy decreased in proportion to a decrease in lesion size. ${ }^{15}$ Similarly, the diagnostic accuracy of CTguided percutaneous lung biopsy in lung 
nodules $\leq 1.5 \mathrm{~cm}$ was lower than that in nodules $>1.5 \mathrm{~cm}^{16}$ In this current study, the detection rate was $100 \%$. The diagnostic rate of malignancy was $76 \%$ (29 of 38), of which 20 of 25 patients were in the group initially diagnosed with unconfirmed lesions and nine of 13 patients were in the group of treated patients that needed additional pathological analyses, which was slightly higher than recent literature reports. ${ }^{12}$ Although lesion size showed no effect on puncture accuracy, $\Delta \mathrm{H}$ tended to decrease with increasing lesion size. Additionally, pulmonary puncture location was found to be a risk factor for puncture angle accuracy $(P=0.033)$. This may have been caused by the difference in respiratory mobilities between different lung lobes, which affected the operator's control of the puncture direction. In general, however, the deviation was well controlled within $10^{\circ}$. A previous study reported that a 3dimensional printed planar template could achieve better unbiased needle insertion. ${ }^{12}$ Nevertheless, this required multiple invasive fixation needles during the operation and lacked the advantages of the frequency of CT scans and operation duration. ${ }^{12}$ In contrast, the method applied in the present study has simplified the puncture process and improved the success rate on the basis of ensuring puncture accuracy, with advantages in puncture efficiency and the frequency of CT scans compared with the 36 CT scans reported to be required in previous studies. ${ }^{12,17}$

The most common complication of pulmonary puncture reported is pneumothorax, followed by haemorrhage, but there is no consensus on the incidence rates of these complications. For example, according to several studies that reported on large sample sizes in recent years, ${ }^{18-22}$ pneumothorax rates range from $3.1 \%$ to $49.3 \%$ and the incidence of haemorrhage was $6.9 \%-29.1 \%$ after CT-guided biopsy. Similarly, other studies have shown pneumothorax rates between $5.3 \%$ and $42 \%$, of which the incidence of the requirement for closed thoracic drainage was between $2 \%$ and $21 \% .^{23-26}$ The incidence of pulmonary haemorrhage was $5 \%-30 \%$ and severe pulmonary haemorrhage occurred in roughly $3.1 \%$ of cases. ${ }^{23-26}$ In this current study, the incidence rate of minor pneumothorax was $36 \%$ and only $6 \%$ progressed to massive pneumothorax. Only three patients suffered needle track bleeding and no other rare complications were observed. The complication rates were consistent with previous studies. ${ }^{18-26}$ Pulmonary haemorrhage seemed to occur less often than the reported rates, ${ }^{23-26}$ possibly due to the improvement of positioning accuracy and patients' comfort in this method. A high one-time success rate also helped to avoid lung tissue and vessel damage caused by repeated needle adjustment. Some researchers have reported that small lesions and repeated puncture had prognostic value for pneumothorax. ${ }^{27} \mathrm{~A}$ previous study reported that pneumothorax was associated with lesion size and the depth of needle penetration. ${ }^{28}$ This current investigation revealed that puncture angle was the main risk factor for pneumothorax, while sex, age, lesion location, lesion size and puncture depth had no effect. This might be related to an insufficient sample size of this study. For this current cohort, the occurrence of complications was less affected by clinically relevant factors, which reflected the safety of this technology to some extent. Yet, these results still need to be verified in a larger research cohort in the future.

This current study had several limitations. First, although the 38 eligible patients that were enrolled in the study were evaluated as thoroughly as possible, the sample size was small. A more comprehensive analysis was limited by this small sample size, particularly when identifying risk factors of puncture accuracy and complications. For small lesions with high puncture difficulty, it would be more convincing if a subgroup 
analysis was performed on a larger sample size to verify the technical superiority of this method relative to the current standard of care. Secondly, the number of patients that underwent abdominal biopsy was very limited, making it hard to explore more details in this subgroup. Further investigation in different tissues is required.

In conclusion, this current study summarized the initial experience and results of the clinical application of a TPS-assisted largeaperture CT simulator-guided percutaneous biopsy to improve the biopsy procedure by combining the advantages of radiotherapy specialties. This emerging technology may enhance the puncture accuracy and success rate under the premise of ensuring safety and efficiency, which is worth promoting in clinical applications.

\section{Acknowledgements}

We are grateful to our colleagues from the departments of oncology and surgical oncology that provided expertise that greatly assisted this research. We also express our immense appreciation to Professor Jiade $\mathrm{Lu}$ for sharing his knowledge and his comments on an earlier version of the manuscript.

\section{Declaration of conflicting interest}

The authors declare that there are no conflicts of interest.

\section{Funding}

This research received no specific grant from any funding agency in the public, commercial, or not-for-profit sectors.

\section{ORCID iD}

Xiangquan Kong (D) https://orcid.org/00000003-0606-0234

\section{References}

1. Kinoshita F, Kato T, Sugiura K, et al. CTguided transthoracic needle biopsy using a puncture site-down positioning technique. AJR Am J Roentgenol 2006; 187: 926-932.
2. Helck A, Schumann C, Aumann J, et al. Automatic path proposal computation for CT-guided percutaneous liver biopsy. Int $J$ Comput Assist Radiol Surg 2016; 11: 2199-2205.

3. Lalji UC, Wildberger JE, Zur Hausen A, et al. CT-guided percutaneous transthoracic needle biopsies using $10 \mathrm{G}$ large-core needles: initial experience. Cardiovasc Intervent Radiol 2015; 38: 1603-1610.

4. Aktas AR, Gozlek E, Yilmaz O, et al. CT-guided transthoracic biopsy: histopathologic results and complication rates. Diagn Interv Radiol 2015; 21: 67-70.

5. Lang D, Reinelt V, Horner A, et al. Complications of CT-guided transthoracic lung biopsy: A short report on current literature and a case of systemic air embolism. Wien Klin Wochenschr 2018; 130: 288-292.

6. Lee HY and Lee IJ. Assessment of independent risk factors of developing pneumothorax during percutaneous core needle lung biopsy: focus on lesion depth. Iran J Radiol 2016; 13: e30929.

7. Nour-Eldin NE, Alsubhi M, Emam A, et al. Pneumothorax complicating coaxial and non-coaxial CT-guided lung biopsy: comparative analysis of determining risk factors and management of pneumothorax in a retrospective review of 650 patients. Cardiovasc Intervent Radiol 2016; 39: 261-270.

8. Reck M and Rabe KF. Precision diagnosis and treatment for advanced non-small-cell lung cancer. $N$ Engl J Med 2017; 377: 849-861.

9. Kaul KL. Preparing pathology for precision medicine: challenges and opportunities. Virchows Arch 2017; 471: 141-146.

10. Dong J, Li B, Lin D, et al. Advances in targeted therapy and immunotherapy for non-small cell lung cancer based on accurate molecular typing. Front Pharmacol 2019; 10: 230 .

11. Russo U, Sabatino V, Nizzoli R, et al. Transthoracic computed tomographyguided lung biopsy in the new era of personalized medicine. Future Oncol 2019; 15: $1125-1134$.

12. Ji Z, Wang G, Chen B, et al. Clinical application of planar puncture template-assisted computed tomography-guided percutaneous 
biopsy for small pulmonary nodules. $J$ Cancer Res Ther 2018; 14: 1632-1637.

13. Fior D, Vacirca F, Leni D, et al. Virtual guidance of percutaneous transthoracic needle biopsy with $\mathrm{C}$-arm cone-beam CT: diagnostic accuracy, risk factors and effective radiation dose. Cardiovasc Intervent Radiol 2019; 42: 712-719.

14. Appelbaum L, Sosna J, Nissenbaum Y, et al. Electromagnetic navigation system for CTguided biopsy of small lesions. AJR Am J Roentgenol 2011; 196: 1194-1200.

15. Tsukada H, Satou T, Iwashima A, et al. Diagnostic accuracy of CT-guided automated needle biopsy of lung nodules. AJR Am J Roentgenol 2000; 175: 239-243.

16. Kothary N, Lock L, Sze DY, et al. Computed tomography-guided percutaneous needle biopsy of pulmonary nodules: impact of nodule size on diagnostic accuracy. Clin Lung Cancer 2009; 10: 360-363.

17. Grasso RF, Faiella E, Luppi G, et al. Percutaneous lung biopsy: comparison between an augmented reality CT navigation system and standard CT-guided technique. Int J Comput Assist Radiol Surg 2013; 8: 837-848.

18. Guimaraes MD, Marchiori E, Hochhegger $\mathrm{B}$, et al. CT-guided biopsy of lung lesions: defining the best needle option for a specific diagnosis. Clinics (Sao Paulo) 2014; 69: 335-340.

19. Yang W, Sun W, Li Q, et al. Diagnostic accuracy of CT-guided transthoracic needle biopsy for solitary pulmonary nodules. PLoS One 2015; 10: e0131373.

20. Otto S, Mensel B, Friedrich N, et al. Predictors of technical success and rate of complications of image-guided percutaneous transthoracic lung needle biopsy of pulmonary tumors. PLoS One 2015; 10: e0124947.
21. Wang W, Yu L, Wang Y, et al. Radial EBUS versus CT-guided needle biopsy for evaluation of solitary pulmonary nodules. Oncotarget 2018; 9: 15122-15131.

22. Rotolo N, Floridi C, Imperatori A, et al. Comparison of cone-beam CT-guided and CT fluoroscopy-guided transthoracic needle biopsy of lung nodules. Eur Radiol 2016; 26: 381-389.

23. Yildirim E, Kirbas I, Harman A, et al. CTguided cutting needle lung biopsy using modified coaxial technique: factors effecting risk of complications. Eur J Radiol 2009; 70: 57-60.

24. Yeow KM, See LC, Lui KW, et al. Risk factors for pneumothorax and bleeding after CT-guided percutaneous coaxial cutting needle biopsy of lung lesions. $J$ Vasc Interv Radiol 2001; 12: 1305-1312.

25. Fontaine-Delaruelle C, Souquet PJ, Gamondes D, et al. Predictive factors of complications during CT-guided transthoracic biopsy. Rev Pneumol Clin 2017; 73: 61-67 [Article in French, English abstract].

26. Dai WR, Li L, Li X, et al. Complications and influencing factors of pneumoconiosis patients undergoing $\mathrm{CT}$ guided percutaneous lung biopsy. Zhonghua Lao Dong Wei Sheng Zhi Ye Bing Za Zhi 2019; 37: 56-60 [Article in Chinese, English abstract].

27. $\mathrm{Wu} \mathrm{CC}$, Maher MM and Shepard JA. Complications of CT-guided percutaneous needle biopsy of the chest: prevention and management. AJR Am J Roentgenol 2011; 196: W678-W682.

28. Yin ZY, Lin ZY, Wang Y, et al. Risk factors of complications after CT-guided percutaneous needle biopsy of lumps near pulmonary hilum. J Huazhong Univ Sci Technol Med Sci 2015; 35: 278-282. 